\title{
Bilipschitz embedding of Grushin plane in $\mathbb{R}^{3}$
}

\author{
JANG-MEI WU
}

Abstract. The Grushin plane is bilipschitz homeomorphic to a quasiplane in $\mathbb{R}^{3}$.

Mathematics Subject Classification (2010): 53C17 (primary); 30L05 (secondary).

\section{Grushin plane}

The Grushin plane $\mathbb{G}$ is the space $\mathbb{R}^{2}$ endowed with the vector fields

$$
X_{1}=\partial_{x_{1}} \quad \text { and } \quad X_{2}=x_{1} \partial_{x_{2}}
$$

Outside the singular line $x_{1}=0$, the Grushin metric is the Riemannian metric

$$
d s^{2}=d x_{1}^{2}+x_{1}^{-2} d x_{2}^{2},
$$

which makes $X_{1}, X_{2}$ an orthonormal basis for the tangent space at each point not on the singular line. The vector field $\left[X_{1}, X_{2}\right]=\partial_{x_{2}}$ is added to the singular line; and the Grushin metric extended across $x_{1}=0$ is the Carnot-Carathéodory metric

$$
d_{\mathbb{G}}(p, q)=\inf _{\gamma} \int_{0}^{1} \sqrt{x_{1}^{\prime}(t)^{2}+\frac{x_{2}^{\prime}(t)^{2}}{x_{1}(t)^{2}}} d t \quad \forall p, q \in \mathbb{R}^{2},
$$

where the infimum is taken over all paths $\gamma:[0,1] \rightarrow \mathbb{G}$ connecting $\gamma(0)=p$ to $\gamma(1)=q$, that are absolutely continuous with respect to the Euclidean metric. On the singular line, $d_{\mathbb{G}}\left(\left(0, x_{2}\right),\left(0, y_{2}\right)\right) \simeq \sqrt{\left|x_{2}-y_{2}\right|}$.

The Grushin plane is in some sense one of the simplest singular sub-Riemannian manifolds. For geodesics in $\mathbb{G}$ and properties of $\mathbb{G}$, see Bellac̈he [2].

The Grushin balls $B_{\mathbb{G}}(x, r)$ can be described in terms of Euclidean rectangles

$$
R(x, r)=\left[x_{1}-r, x_{1}+r\right] \times\left[x_{2}-r\left(\left|x_{1}\right|+r\right), x_{2}+r\left(\left|x_{1}\right|+r\right)\right] .
$$

This work was supported in part by the National Science Foundation grant DMS-1001669.

Received August 10, 2012; accepted in revised version May 3, 2013. 
Precisely, there is a constant $C>1$ such that $R\left(x, C^{-1} r\right) \subset B_{\mathbb{G}}(x, r) \subset R(x, C r)$ for all $x=\left(x_{1}, x_{2}\right) \in \mathbb{R}^{2}$ and $r>0$; see Franchi and Lanconelli [5], also [4]. Therefore outside the line $x_{1}=0$, the Grushin plane has a Whitney-type decomposition

$$
\begin{aligned}
& \mathbb{G} \backslash\left\{x_{1}=0\right\}=\bigcup_{k \in \mathbb{Z}} \bigcup_{m \in \mathbb{Z}}( {\left[7^{k-1}, 7^{k}\right] \times\left[m 49^{k},(m+1) 49^{k}\right] \cup\left[-7^{k},-7^{k-1}\right] } \\
&\left.\times\left[m 49^{k},(m+1) 49^{k}\right]\right) .
\end{aligned}
$$

Meyerson [6] has shown that the Grushin plane is quasisymmetrically equivalent to the Euclidean plane by the map $\left(x_{1}, x_{2}\right) \mapsto\left(x_{1}\left|x_{1}\right|, x_{2}\right)$. Seo [9] has proved a general theorem on bilipschitz embeddable metric spaces, from which it follows that the Grushin plane can be bilipschitzly embedded into some Euclidean space.

Since the number of Grushin balls of radius $\varepsilon>0$ needed to cover the unit ball $B_{\mathbb{G}}((0,0), 1)$ has magnitude $\asymp \varepsilon^{-2} \log \frac{1}{\varepsilon}$ for small $\varepsilon$ [2], the Grushin plane can not be bilipschitzly embedded into $\mathbb{R}^{2}$. On the other hand it is relatively simple to embed $\mathbb{G}$ bilipschitzly into $\mathbb{R}^{4}$.

We prove a sharp embedding theorem for the Grushin plane.

Theorem 1.1. The Grushin plane $\mathbb{G}$ is bilipschitz homeomorphic to a quasiplane in $\mathbb{R}^{3}$.

The embedded singular line in $\mathbb{R}^{3}$ is necessarily a very regular snowflake curve $\Gamma$ of Hausdorff dimension 2. The goal is to place, in a bounded Euclidean neighborhood of a subarc of the embedded $\Gamma, 49^{k}$ wrinkled Whitney 2-cells of diameter comparable to $7^{-k}$ for all $k \geq 0$.

In contrast, Semmes [8] has observed that the first Heisenberg group $\mathbb{H}$, when equipped with its Carnot metric, can not be bilipschitzly embedded into any $\mathrm{Eu}-$ clidean space $\mathbb{R}^{n}$. Semmes' observation is based on a deep theorem of Pansu [7].

A quasiplane in $\mathbb{R}^{3}$ is the image of the hyperplane $\mathbb{R}^{2}$ in $\mathbb{R}^{3}$ under a global quasiconformal homeomorphism of $\mathbb{R}^{3}$.

A sense-preserving homeomorphism $f: D \rightarrow D^{\prime}$ between domains in $\mathbb{R}^{n}, n \geq$ 2 , is said to be quasiconformal if

$$
\limsup _{r \rightarrow 0} \frac{\max \{|f(y)-f(x)|:|y-x|=r\}}{\min \{|f(y)-f(x)|:|y-x|=r\}} \leq H<\infty
$$

for all $x \in D$ and some $H$ independent of $x$. This is the so-called metric definition of quasiconformal maps. For the connection between this and the geometrical or the analytic definition of quasiconformal maps, see the book by Väisälä [10].

A mapping $f:\left(X, d_{X}\right) \rightarrow\left(Y, d_{Y}\right)$ between metric spaces is said to be $L$ bilipschitz if

$$
d_{X}(x, y) / L \leq d_{Y}(f(x), f(y)) \leq L d_{X}(x, y)
$$

for all $x, y \in X$ and some constant $L \geq 1$; and a mapping is a $(\lambda, L)$-quasisimilarity if

$$
\frac{\lambda}{L} d_{X}(x, y) \leq d_{Y}(f(x), f(y)) \leq \lambda L d_{X}(x, y)
$$


for all $x, y \in X$ and some constants $L \geq 1$ and $\lambda>0$. Clearly (1, $L$ )-quasisimilarities are the $L$-bilipschitz mappings, $(\lambda, 1)$-quasisimilarities are similarities with scaling factor $\lambda$, and 1-similarities are isometries.

\section{Building components}

The construction in this section is largely based on an example of Assouad in [1] and an example of Bonk and Heinonen in [3]. The book-keeping below is needed in the eventual bilipschitz estimates.

\subsection{Blocks}

Let $\mathcal{C}$ be the cube $\left[-\frac{7}{2}, \frac{7}{2}\right] \times\left[-\frac{7}{2}, \frac{7}{2}\right] \times[0,7], I$ be the (directed) line segment from $(0,0,0)$ to $(0,0,7)$, and

$$
Q_{I}=[-3,3] \times[-3,3] \times[0,7],
$$

a rectangular cube in $\mathcal{C}$ that meets $\partial \mathcal{C}$ in two squares. We call $Q_{I}$ an $I$-block, squares $Q_{I} \cap\left\{x_{3}=0\right\}$ and $Q_{I} \cap\left\{x_{3}=7\right\}$ the entrance and the exit of $Q_{I}$ respectively, and $\partial Q_{I}$ minus the union of the interiors of the entrance and the exit the side $s Q_{I}$ of $Q_{I}$.

Let $L$ be the (directed) line segment from $(0,0,0)$ to $\left(0,0, \frac{7}{2}\right)$ followed by the (directed) line segment from $\left(0,0, \frac{7}{2}\right)$ and $\left(0, \frac{7}{2}, \frac{7}{2}\right)$, and

$$
Q_{L}=\left([-3,3] \times[-3,3] \times\left[0, \frac{13}{2}\right]\right) \cup\left([-3,3] \times\left[-3, \frac{7}{2}\right] \times\left[\frac{1}{2}, \frac{13}{2}\right]\right)
$$

be a union of two rectangular cubes in $\mathcal{C}$ that meets $\mathcal{C}$ in two squares. We call $Q_{L}$ an $L$-block, squares $Q_{L} \cap\left\{x_{3}=0\right\}$ and $Q_{L} \cap\left\{x_{2}=\frac{7}{2}\right\}$ the entrance and the exit of $Q_{L}$ respectively, and the boundary $\partial Q_{L}$ minus the union of the interiors of the entrance and the exit the side $s Q_{L}$ of $Q_{L}$.

We write $Q$ for $Q_{I}$ or $Q_{L}$ when the type of the block is inessential. Images of $I, L, Q_{I}$, or $Q_{L}$ under similarities are again called $I$-segments, $L$-segments, $I$ blocks, or $L$-blocks; they have a naturally inherited direction. When $h$ is a similarity map and $\ell$ is either the $I$-segment $h(I)$ or the $L$-segment $h(L)$, we write $Q_{\ell}$ for $h\left(Q_{I}\right)$ or $h\left(Q_{L}\right)$, and call $Q_{\ell}$ the block associated with the segment $\ell$.

\subsection{Cores}

There exists a simple polygonal path $J_{I}$ in $Q_{I}$ going from $(0,0,0)$ to $(0,0,7)$, which is symmetric with respect to the plane $x_{3}=\frac{7}{2}$ and has the following properties:

(1) $J_{I}$ is unknotted in $Q_{I}$, in the sense that there is a homeomorphism of $Q_{I}$ onto itself which is the identity map on $\partial Q_{I}$ and maps $J_{I}$ onto the line segment from $(0,0,0)$ to $(0,0,7)$. 
(2) $J_{I}$ consists of a total of $49 I$-segments and $L$-segments, $\ell_{1}, \ldots, \ell_{49}$, of unit length, with mutually disjoint interiors, and labeled according to their order in $J_{I}$. Moreover, segments $\ell_{1}, \ell_{49}$ and at least one other are $I$-segments.

(3) All blocks $Q_{\ell_{m}}$ are contained in the interior of $Q_{I}$ with the exception that $Q_{\ell_{1}} \cap \partial Q_{I}$ is the entrance of $Q_{\ell_{1}}$ and $Q_{\ell_{49}} \cap \partial Q_{I}$ is the exit of $Q_{\ell_{49}}$. Only blocks associated to the consecutive segments meet, and they meet in such a way that $Q_{\ell_{m}} \cap Q_{\ell_{m+1}}$ is the exit of $Q_{\ell_{m}}$ and the entrance of $Q_{\ell_{m+1}}$. Finally, the union $\bigcup_{1}^{49} Q_{\ell_{m}}$ of the blocks is homeomorphic to a cube.

We call $\kappa_{Q_{I}}:=\bigcup_{1}^{49} Q_{\ell_{m}}$ the core of $Q_{I}$. The entrance, the exit and the side of the core are canonically defined. For simplicity, we will write $Q_{m}$ for $Q_{\ell_{m}}$.

Similarly, there is a simple polygonal path $J_{L}$ in $Q_{L}$ going from $(0,0,0)$ to $\left(0, \frac{7}{2}, \frac{7}{2}\right)$, which is symmetric with respect to the plane $x_{3}=\frac{7}{2}-x_{2}$ and satisfies the analogues of (1), (2) and (3) adapted for $Q_{L}$. Here, only segments $\ell_{1}$ and $\ell_{49}$ are required to be $I$-segments. The core $\kappa_{Q_{J}}$ of $Q_{J}$, and the entrance, the exit and the side of the core are defined analogously. (Examples of paths $J_{I}$ and $J_{L}$ are given in the appendix.)

Symmetry of the paths is imposed to simplify the argument below; it is not essential. There is nothing special about the numbers 7 and 49 either, except that $\frac{\log 49}{\log 7}$ is the Hausdorff dimension of the singular line in $\mathbb{G}$.

\subsection{Edges}

Block $Q_{I}$ has four edges on its side, namely, $\{(3,3, t): 0 \leq t \leq 7\},\{(-3,3, t): 0 \leq$ $t \leq 7\},\{(-3,-3, t): 0 \leq t \leq 7\}$, and $\{(3,-3, t): 0 \leq t \leq 7\}$; only one will be labeled

$$
e_{0}=\{(3,3, t): 0 \leq t \leq 7\}
$$

Block $Q_{L}$ has four edges on its side; they are labeled as

$$
\begin{aligned}
& e_{1}=\left\{(3,3, t): 0 \leq t \leq \frac{1}{2}\right\} \cup\left\{\left(3, t, \frac{1}{2}\right): 3 \leq t \leq \frac{7}{2}\right\}, \\
& e_{2}=\left\{(-3,3, t): 0 \leq t \leq \frac{1}{2}\right\} \cup\left\{\left(-3, t, \frac{1}{2}\right): 3 \leq t \leq \frac{7}{2}\right\}, \\
& e_{3}=\left\{(-3,-3, t): 0 \leq t \leq \frac{13}{2}\right\} \cup\left\{\left(-3, t, \frac{13}{2}\right):-3 \leq t \leq \frac{7}{2}\right\}, \\
& e_{4}=\left\{(3,-3, t): 0 \leq t \leq \frac{13}{2}\right\} \cup\left\{\left(3, t, \frac{13}{2}\right):-3 \leq t \leq \frac{7}{2}\right\} .
\end{aligned}
$$

We define edge paths along core $\kappa_{Q_{L}}$ as follows. An edge path initiates at a vertex $p$ of $\kappa_{Q_{L}}$ on the plane $x_{3}=0$, it moves along the edge of the first block in $\kappa_{Q_{L}}$ that starts at $p$. When the path reaches the end point $q$ of that edge, it continues along the edge of the second block in $\kappa_{Q_{L}}$ that starts at $q$, and so on. The path stops when it reaches the plane $x_{2}=\frac{7}{2}$. Along the way, the path is marked by the edges it passes through. 
Corresponding to each starting point $p$, there is a unique edge path. Four choices of the starting point result in four mutually disjoint edge paths $w_{1}, w_{2}, w_{3}$, and $w_{4}$, each of which is symmetric with respect to the plane $x_{3}=\frac{7}{2}-x_{2}$. Edge paths are labeled so that $w_{1}, w_{2}, w_{3}$, and $w_{4}$ start at $\left(\frac{3}{7}, \frac{3}{7}, 0\right),\left(-\frac{3}{7}, \frac{3}{7}, 0\right)$, $\left(-\frac{3}{7},-\frac{3}{7}, 0\right)$, and $\left(\frac{3}{7},-\frac{3}{7}, 0\right)$ respectively, and end at $\left(\frac{3}{7}, \frac{7}{2},-\frac{3}{7}+\frac{7}{2}\right),\left(-\frac{3}{7}, \frac{7}{2},-\frac{3}{7}+\right.$ $\left.\frac{7}{2}\right),\left(-\frac{3}{7}, \frac{7}{2}, \frac{3}{7}+\frac{7}{2}\right)$, and $\left(\frac{3}{7}, \frac{7}{2}, \frac{3}{7}+\frac{7}{2}\right)$ respectively. Note in particular that for every $i=1,2,3$, or 4 , the origin $(0,0,0)$, the starting point of $w_{i}$, and the starting point of edge $e_{i}$ are collinear, and that the same can be said about the point $\left(0, \frac{7}{2}, \frac{7}{2}\right)$ and the terminal points of $w_{i}$ and $e_{i}$. We call $w_{i} \cap Q_{m}$ the marked edge of $Q_{m}$ derived from data $\left(Q_{L}, e_{i}\right)$, for $m=1, \ldots, 49$.

There are four edge paths along the core $\kappa_{Q_{I}}$ as well. We label the edge path going from $\left(\frac{3}{7}, \frac{3}{7}, 0\right)$ to $\left(\frac{3}{7}, \frac{3}{7}, 7\right)$ by $w_{0}$, and call $w_{0} \cap Q_{m}$ the marked edge of $Q_{m}$ derived from data $\left(Q_{I}, e_{0}\right)$ for $m=1, \ldots, 49$.

\subsection{Tubes}

Consider tubes

$$
\tau_{Q_{I}}=\overline{Q_{I} \backslash \kappa_{Q_{I}}} \quad \text { and } \quad \tau_{Q_{L}}=\overline{Q_{L} \backslash \kappa_{Q_{L}}}
$$

obtained by removing the cores from the blocks. The entrance and the exit of $\tau_{Q_{I}}$ are canonically defined; they are congruent to the rectangular annulus

$$
A=([-3,3] \times[-3,3]) \backslash\left(\left(-\frac{3}{7}, \frac{3}{7}\right) \times\left(-\frac{3}{7}, \frac{3}{7}\right)\right) \subset \mathbb{R}^{2} \times\{0\} .
$$

The remaining part of $\partial \tau_{Q_{I}}$ is composed of the side $s Q_{I}$ of block $Q_{I}$ and the side $s \kappa_{Q_{I}}$ of core $\kappa_{Q_{I}}$. The boundary of $\tau_{Q_{L}}$ can be similarly partitioned.

\subsection{Bilipschitz maps between tubes}

Let $\mathrm{Q}=\left\{x \in \mathbb{R}^{2}:|x| \leq 1\right\} \times[0,1]$ be a round block in $\mathbb{R}^{3}$ having a core $\mathrm{k}=$ $\left\{x \in \mathbb{R}^{2}:|x| \leq \frac{1}{49}\right\} \times[0,1]$, that is composed of 49 congruent blocks $Q_{m}=$ $\left\{x \in \mathbb{R}^{2}:|x| \leq \frac{1}{49}\right\} \times\left[\frac{m-1}{49}, \frac{m}{49}\right], m=1, \ldots, 49$. Denote by $\mathrm{t}=\overline{\mathrm{Q} \backslash \mathrm{k}}$ the round tube $\left\{x \in \mathbb{R}^{2}: \frac{1}{49} \leq|x| \leq 1\right\} \times[0,1]$, and by $\mathrm{t}_{m}=\left\{x \in \mathbb{R}^{2}: \frac{1}{49^{2}} \leq|x| \leq\right.$ $\left.\frac{1}{49}\right\} \times\left[\frac{m-1}{49}, \frac{m}{49}\right], m=1, \ldots, 49,49$ congruent tubes.

Denote by $\mathrm{e}=\{(1,0, t): 0 \leq t \leq 1\}$ an edge of $\mathrm{Q}$, and by $\mathrm{w}=\left\{\left(\frac{1}{49}, 0, t\right): 0 \leq\right.$ $t \leq 1\}$ an edge path along $\mathrm{k}$; let $\mathrm{A}=\left\{x \in \mathbb{R}^{2}: \frac{1}{49} \leq|x| \leq 1\right\}$ be the annulus in $\mathbb{R}^{2} \times\{0\}$ that is congruent to the entrance and the exit of the tube $t$.

Define an isometric involution in each of $Q_{I}, Q_{L}$, and $\mathrm{Q}$, by the reflection with respect to the planes $x_{3}=\frac{7}{2}, x_{3}=\frac{7}{2}-x_{2}$, or $x_{3}=\frac{1}{2}$ respectively.

Let $\zeta_{m}$ be the similarity map in $\mathbb{R}^{3}$ with

$$
\zeta_{m}:(\mathrm{Q}, \mathrm{e}) \rightarrow\left(\mathrm{Q}_{m}, \mathrm{w} \cap \mathrm{Q}_{m}\right),
$$


and $\varrho_{\alpha}$ be the rotation of $\mathbb{R}^{2}$ about the origin by an angle $\alpha$.

Let $\phi: \mathrm{A} \rightarrow A$ be the sense-preserving homeomorphism from the annulus $\mathrm{A}$ onto the rectangular annulus $A$, that maps each radial segment in A linearly onto a radial segment in $A$ having the same argument. Let $\varphi=\phi \circ \varrho_{\pi / 4}$. Note that $\varphi$ maps the points $(1,0),(0,1),(-1,0),(0,-1)$ to the points $(3,3),(-3,3),(-3,-3)$, $(3,-3)$, respectively.

Let $\theta_{0}:(s \mathrm{Q}, \mathrm{e}) \rightarrow\left(s Q_{I}, e_{0}\right)$ be the homeomorphism

$$
\theta_{0}\left(x_{1}, x_{2}, x_{3}\right)=\left(\varphi\left(x_{1}, x_{2}\right), x_{3}\right)
$$

fix, for each $i \in\{1,2,3,4\}$, a bilipschitz homeomorphism $\theta_{i}:(s \mathrm{Q}, \mathrm{e}) \rightarrow\left(s Q_{L}, e_{i}\right)$ that satisfies

$$
\theta_{i}\left(x_{1}, x_{2}, 0\right)=\left(\varphi \circ \varrho_{(i-1) \pi / 2}\left(x_{1}, x_{2}\right), 0\right)
$$

and intertwines the involutions in $\mathrm{Q}$ and $Q_{L}$.

Given an integer $i \in\{0,1,2,3,4\}$, set $Q=Q_{I}$ when $i=0$, and $Q=$ $Q_{L}$ when $i \neq 0$. We will define, as in [3], a collection of five basic bilipschitz homeomorphisms $\Theta_{i}:(\mathrm{t}, \mathrm{e}, \mathrm{w}) \rightarrow\left(\tau_{Q}, e_{i}, w_{i}\right), i=0, \ldots, 4$.

We first define a bilipschitz homeomorphism $\vartheta_{i}:(\partial \mathrm{t}, \mathrm{e}, \mathrm{w}) \rightarrow\left(\partial \tau_{Q}, e_{i}, w_{i}\right)$ on the boundary following these steps:

(i) on the outer side of $\mathrm{t}$, mapping $\vartheta_{i} \mid s \mathrm{Q}=\theta_{i}:(s \mathrm{Q}, \mathrm{e}) \rightarrow\left(s Q, e_{i}\right)$;

(ii) the restriction of $\vartheta_{i}$ to the entrance (or the exit) of $t$ is $\varphi$ modulo an isometry;

(iii) associated to each block $Q_{m}$ in the core $\kappa_{Q}$, there is a marked edge $\varepsilon\left(Q_{m}\right)=$ $w_{i} \cap Q_{m}$ derived from $\left(Q, e_{i}\right)$, hence there exist a unique $Q\left(Q_{m}\right) \in\left\{Q_{I}, Q_{L}\right\}$, a unique $\iota\left(Q_{m}\right) \in\{0,1,2,3,4\}$, and a similarity map $\sigma_{m}:\left(Q\left(Q_{m}\right), e_{\iota}\left(Q_{m}\right)\right) \rightarrow$ $\left(Q_{m}, \varepsilon\left(Q_{m}\right)\right)$. (To ease the notations, the dependency of $i$ is not recorded.) The inner side of $\mathrm{t}$ is $s \mathrm{k}$; the mapping $\vartheta_{i} \mid s \mathrm{k}:(s \mathrm{k}, \mathrm{w}) \rightarrow\left(s \kappa_{Q}, w_{i}\right)$ is defined by gluing together the maps $\vartheta_{i} \mid s \mathbf{Q}_{m}=\sigma_{m} \circ \theta_{\iota\left(Q_{m}\right)} \circ \zeta_{m}^{-1}, 1 \leq m \leq 49$. The gluing is well-defined because the union of marked edges of $Q_{m}$ 's is the edge path $w_{i}$.

During this process, each block $Q_{m}$ inherits from $\left(Q\left(Q_{m}\right), e_{\iota(} Q_{m}\right)$ a core and a marked edge on each of the 49 blocks in this core, via the similarity map $\sigma_{m}$.

Since $J_{I}\left(\right.$ or $J_{L}$ ) is unknotted in $Q$ and $\kappa_{Q}$ is a regular neighborhood of $J_{I}$ (or $\left.J_{L}\right)$ in $Q$, there is a (bilipschitz) homeomorphism $\Phi:\left(Q, \kappa_{Q}, e_{i}\right) \rightarrow(\mathrm{Q}, \mathrm{k}, \mathrm{e})$ that agrees with $\vartheta_{i}^{-1}$ on $\partial Q \backslash \kappa_{Q}$ and respects the given involutions. Since $w_{i}\left(\subset \partial \kappa_{Q}\right)$ is fixed by the involution in $Q, \Phi\left(w_{i}\right)(\subset \mathrm{Q})$ is symmetric with respect to the plane $x_{3}=\frac{1}{2}$. Therefore $\Phi\left(w_{i}\right)$ can be straightened so that $\Phi\left(w_{i}\right)=\mathrm{w}$. The composition $\Phi \circ \vartheta_{i}:(\partial \mathrm{t}, \mathrm{e}, \mathrm{w}) \rightarrow(\partial \mathrm{t}, \mathrm{e}, \mathrm{w})$, which is identity on $\partial \mathrm{t} \backslash \mathrm{k}$, clearly has a bilipschitz extension $\Psi: \mathrm{t} \rightarrow \mathrm{t}$. Therefore $\vartheta_{i} \mid \partial \mathrm{t}$ has a bilipschitz extension

$$
\Theta_{i}=\Phi^{-1} \circ \Psi:(\mathrm{t}, \mathrm{e}, \mathrm{w}) \rightarrow\left(\tau_{Q}, e_{i}, w_{i}\right)
$$

between tubes. 
We now have five basic bilipschitz homeomorphisms $\Theta_{i}:(\mathrm{t}, \mathrm{e}, \mathrm{w}) \rightarrow\left(\tau_{Q}, e_{i}, w_{i}\right)$, $i=0, \ldots, 4$, at our disposal. Uniqueness has been emphasized throughout their construction to ensure the forward and backward iteration processes below are selfsimilar.

\subsection{Quasiconformal maps between blocks}

We define a quasiconformal map $f$ from block $\mathrm{Q}$ onto block $Q_{I}$ with data $\left(Q_{I}, e_{0}\right)$. Set

$$
\mathrm{K}_{-k}=\left\{x \in \mathbb{R}^{2}:|x| \leq 49^{-k}\right\} \times[0,1] \text { and } \mathrm{T}_{-k}=\overline{\mathrm{K}_{-k} \backslash \mathrm{K}_{-k-1}},
$$

for all $k \geq 0$. Then

$$
\mathrm{Q}=\{(0,0, t): 0 \leq t \leq 1\} \cup \bigcup_{k \geq 0} \mathrm{~T}_{-k}
$$

Set also $Q=Q_{I}, K_{0}=Q, K_{-1}=\kappa_{Q}$, and $T_{0}=\overline{K_{0} \backslash K_{-1}}=\tau_{Q}$; note that $\mathrm{T}_{0}=\mathrm{t}$, and let

$$
f \mid \mathrm{T}_{0}=\Theta_{0}: \mathrm{T}_{0} \rightarrow T_{0} .
$$

For every $m \in[1,49]$, let $\varepsilon\left(Q_{m}\right)$ be the marked edge on $Q_{m}$ derived from $\left(Q_{I}, e_{0}\right)$, and $\sigma_{m}:\left(Q\left(Q_{m}\right), e_{l\left(Q_{m}\right)}\right) \rightarrow\left(Q_{m}, \varepsilon\left(Q_{m}\right)\right)$ be the similarity used in constructing $\Theta_{0}$. The similarity $\sigma_{m}$ induces naturally a core $\kappa_{m}\left(=\kappa_{Q_{m}}\right)$, consequently a tube $\tau_{m}=\overline{Q_{m} \backslash \kappa_{m}}$ to each block $Q_{m}$ in $K_{-1}$.

Set $K_{-2}=\cup_{m} \kappa_{m}$ and $T_{-1}=\overline{K_{-1} \backslash K_{-2}}=\cup_{m} \tau_{Q_{m}}$. Since $\mathrm{T}_{-1}=\cup_{m} \mathrm{t}_{m}$, the mapping $f \mid \mathrm{T}_{-1}: \mathrm{T}_{-1} \rightarrow T_{-1}$ will be defined by gluing together homeomorphisms

$$
f \mid \mathbf{t}_{m}=\sigma_{m} \circ \Theta_{\iota\left(Q_{m}\right)} \circ \zeta_{m}^{-1}: \mathbf{t}_{m} \rightarrow \tau_{Q_{m}} .
$$

Because the union $W_{-1}$ of marked edges $\varepsilon\left(Q_{m}\right)$ is an edge path along $\kappa_{Q}$ going from $\left(\frac{3}{7}, \frac{3}{7}, 0\right)$ to $\left(\frac{3}{7}, \frac{3}{7}, 7\right)$, and the restrictions of $f \mid t_{m}$ to the entrance and to the exit of $\mathrm{t}_{m}$ are essentially identical (modulo isometries) for all $m$, we conclude that the gluing, therefore the homeomorphism $f \mid \mathrm{T}_{-1}$, is well-defined. We now have the extension

$$
f: \mathrm{T}_{0} \cup \mathrm{T}_{-1} \rightarrow T_{0} \cup T_{-1} .
$$

Before starting the next step, we write $\varepsilon_{m}, \iota_{m}, Q(m)$ in place of $\varepsilon\left(Q_{m}\right), \iota\left(Q_{m}\right)$, $Q\left(Q_{m}\right)$ to simplify the notation, and replace the index $m$ in the previous step by $m_{1}$. For each $m_{1} \in[1,49]$, the process of defining $f \mid \mathrm{t}_{m_{1}}$ has uniquely defined a core $\kappa_{m_{1}, m_{2}}$, a tube $\tau_{m_{1}, m_{2}}$, a marked edge $\varepsilon_{m_{1}, m_{2}}$, a block $Q\left(m_{1}, m_{2}\right) \in\left\{Q_{I}, Q_{L}\right\}$, a number $\iota_{m_{1}, m_{2}} \in\{0,1,2,3,4\}$, and a similarity map $\sigma_{m_{1}, m_{2}}:\left(Q\left(m_{1}, m_{2}\right), e_{\iota_{m_{1}, m_{2}}}\right) \rightarrow$ $\left(Q_{m_{1}, m_{2}}, \varepsilon_{m_{1}, m_{2}}\right)$, associated to each of the $49\left(1 \leq m_{2} \leq 49\right)$ blocks $Q_{m_{1}, m_{2}}$ in the core $\kappa_{m_{1}}$.

The union $W_{-2}$ of these $49^{2}$ marked edges is an edge path along $K_{-2}$ from $\left(\frac{3}{7^{2}}, \frac{3}{7^{2}}, 0\right)$ to $\left(\frac{3}{7^{2}}, \frac{3}{7^{2}}, 7\right)$, and the union $K_{-3}$ of the cores of these $49^{2}$ new blocks is 
a topological cube. Set $T_{-2}=\overline{K_{-2} \backslash K_{-3}}$. We now extend $f: \mathrm{T}_{0} \rightarrow \mathrm{T}_{-1} \cup \mathrm{T}_{-2} \rightarrow$ $T_{0} \cup T_{-1} \cup T_{-2}$ by gluing together homeomorphisms

$$
f \mid \mathbf{t}_{m_{1}, m_{2}}=\sigma_{m_{1}, m_{2}} \circ \Theta_{l_{m_{1}, m_{2}}} \circ \zeta_{m_{2}}^{-1} \circ \zeta_{m_{1}}^{-1}: \mathbf{t}_{m_{1}, m_{2}} \rightarrow \tau_{m_{1}, m_{2}} .
$$

We observe, after a moment's reflection, the self-similar property on $I$-blocks: whenever $Q_{m_{1}}$ is an $I$-block,

$$
f\left|\mathbf{t}_{m_{1}, m_{2}}=\sigma_{m_{1}} \circ f\right| \mathbf{t}_{m_{2}} \circ \zeta_{m_{1}}^{-1} \mid \mathbf{t}_{m_{1}, m_{2}} .
$$

Continue this process inductively in a self-similar manner, we arrive at a homeomorphism $f$ from $\mathrm{Q} \backslash\{(0,0, t): 0 \leq t \leq 1\}$ onto $Q_{I} \backslash \gamma$, where $\gamma$ is the snowflake $\operatorname{arc}$

$$
\gamma=\bigcap_{k=1}^{\infty} K_{-k} .
$$

In view of the scaling in the domain and in the target, $f$ is a $\left(7^{-k}, C\right)$-quasisimilarity on each of the $49^{k}$ tubes in $T_{-k}$, for some constant $C>1$, therefore the mapping $f: \mathbf{Q} \backslash\{(0,0, t): 0 \leq t \leq 1\} \rightarrow Q_{I} \backslash \gamma$ is quasiconformal.

By a theorem of Väisälä on removable sets [10], $f$ can be extended to be quasiconformal from $\mathrm{Q}$ onto $Q_{I}$.

\section{Quasiconformal homeomorphism of $\mathbb{R}^{3}$}

\subsection{Quasiconformal extension to $\mathbb{R}^{3}$}

Mapping $f: \mathrm{Q} \rightarrow Q_{I}$ will be extended to a quasiconformal homeomorphism of $\mathbb{R}^{3}$ by backward iteration.

We begin with $Q_{I}$ and a fixed $I$-block $Q_{m^{\prime}}$ in its core with $m^{\prime} \neq 1,49$.

Let $\zeta=\zeta_{m^{\prime}}$ be the similarity in $\mathbb{R}^{3}$ that maps $(\mathrm{Q}, \mathrm{e})$ to $\left(\mathrm{Q}_{m^{\prime}}, \mathrm{w} \cap \mathrm{Q}_{m^{\prime}}\right)$, and $\sigma=\sigma_{m^{\prime}}$ be the similarity in $\mathbb{R}^{3}$ that maps $\left(Q_{I}, e_{0}\right)$ to $\left(Q_{m^{\prime}}, w_{0} \cap Q_{m^{\prime}}\right)$ used in constructing $\Theta_{0}$. Note that $\zeta$ has a scaling factor $1 / 49$ and $\sigma$ has a scaling factor $1 / 7$.

Because $m^{\prime} \neq 1,49$, the space $\mathbb{R}^{3}$ is the union of an increasing sequence of I-blocks

$$
\mathbb{R}^{3}=\bigcup_{k \geq 0} \sigma^{-k} Q_{I},
$$

and also can be expressed as the union of an increasing sequence of round blocks

$$
\mathbb{R}^{3}=\bigcup_{k \geq 0} \zeta^{-k} \mathrm{Q}
$$

Observe that these unions are proper subsets of $\mathbb{R}^{3}$ when $m^{\prime}=1$ or 49 . 
Define homeomorphisms $F_{k}: \zeta^{-k} \mathrm{Q} \rightarrow \sigma^{-k} Q_{I}, k \geq 0$, by

$$
F_{k}=\sigma^{-k} \circ f \circ \zeta^{k} \text {. }
$$

In view of the self-similar property on $I$-blocks, we have $f \circ \zeta \mid \mathrm{Q}=\sigma \circ f$. Therefore, $F_{k}\left|\mathrm{Q}=\sigma^{-k} \circ f \circ \zeta^{k}\right| \mathrm{Q}=f$ for all $k \geq 0$, and

$$
F_{k^{\prime}} \mid \zeta^{-k} \mathrm{Q}=F_{k} \quad \text { for all } k^{\prime} \geq k \geq 0 .
$$

The limiting map $F=\lim _{k \rightarrow \infty} F_{k}: \mathbb{R}^{3} \rightarrow \mathbb{R}^{3}$ is well-defined, homeomorphic and quasiconformal.

\subsection{A snowflake curve and a quasiplane}

The snowflake arc $\gamma=\bigcap_{k=1}^{\infty} K_{-k}$ is in fact $F(\{(0,0, t): 0 \leq t \leq 1\})$. Through backward iterations we get an infinite snowflake curve

$$
\Gamma=\lim _{k \rightarrow \infty} \sigma^{-k} \gamma=F(\{(0,0)\} \times \mathbb{R}) .
$$

The plane $P=\mathbb{R} \times\{0\} \times \mathbb{R}$ in $\mathbb{R}^{3}$ has a decomposition

$$
\begin{aligned}
P=(\{(0,0)\} \times \mathbb{R}) \cup \bigcup_{k \in \mathbb{Z}} \bigcup_{m \in \mathbb{Z}}\left(\left[49^{k-1}, 49^{k}\right] \times\{0\} \times\left[m 49^{k},(m+1) 49^{k}\right]\right. \\
\left.\cup\left[-49^{k},-49^{k-1}\right] \times\{0\} \times\left[m 49^{k},(m+1) 49^{k}\right]\right) \\
=(\{(0,0)\} \times \mathbb{R}) \cup \bigcup_{k \in \mathbb{Z}} \bigcup_{m \in \mathbb{Z}} \mathrm{E}_{k, m} \cup \mathrm{E}_{k, m}^{\prime} .
\end{aligned}
$$

Therefore the quasiplane $\mathcal{P}=F(\mathrm{P})$ can be expressed as

$$
\mathcal{P}=\Gamma \cup \bigcup_{k \in \mathbb{Z}} \bigcup_{m \in \mathbb{Z}} F\left(\mathrm{E}_{k, m}\right) \cup F\left(\mathrm{E}_{k, m}^{\prime}\right) .
$$

Denote by $\check{\mathrm{E}}_{k, m}$ (respectively $\check{\mathrm{E}}_{k, m}^{\prime}$ ) the union of those rectangles in the above decomposition of $\mathrm{P}$ which meet $\mathrm{E}_{k, m}$ (respectively $\mathrm{E}_{k, m}^{\prime}$ ). Then $F \mid \check{\mathbf{E}}_{k, m}$ and $F \mid \check{\mathbf{E}}_{k, m}^{\prime}$ are $\left(7^{-k}, C\right)$-quasisimilarities for some constant $C>1$ independent of $k$ and $m$.

\section{Proof of Theorem 1.1}

From now on we use $\left(x_{1}, x_{3}\right)$ for coordinates in $\mathbb{G}$, and recall that the Grushin metric has the dilation property

$$
d_{\mathbb{G}}\left(\left(\lambda x_{1}, \lambda^{2} x_{3}\right),\left(\lambda y_{1}, \lambda^{2} y_{3}\right)\right)=\lambda d_{\mathbb{G}}\left(\left(x_{1}, x_{3}\right),\left(y_{1}, y_{3}\right)\right) \text { for } \lambda>0 .
$$


Furthermore for any $x=\left(x_{1}, x_{3}\right), y=\left(y_{1}, y_{3}\right) \in \mathbb{G}$,

$$
d_{\mathbb{G}}(x, y) \simeq\left|x_{1}-y_{1}\right|+\min \left\{\sqrt{\left|x_{3}-y_{3}\right|}, \frac{\left|x_{3}-y_{3}\right|}{\max \left\{\left|x_{1}\right|,\left|y_{1}\right|\right\}}\right\} .
$$

This estimate is known; it can be deduced from a theorem of Franchi and Lanconelli in [5] (see also Theorem 3 in [4]). In fact, $\max \left\{\left|x_{1}\right|,\left|y_{1}\right|\right\}$ may be replaced by $\min \left\{\left|x_{1}\right|,\left|y_{1}\right|\right\}$ in the above estimate. Here the expression $a \simeq b$ means that $a, b \geq$ 0 and $C^{-1} \leq a / b \leq C$ for a constant $C \geq 1$.

Let $H: \mathbb{G} \rightarrow \mathrm{P}$ be the homeomorphism

$$
H:\left(x_{1}, x_{3}\right) \mapsto\left(x_{1}\left|x_{1}\right|, 0, x_{3}\right) ;
$$

then the composition $F \circ H$ is a homeomorphism from the Grushin plane $\mathbb{G}$ to the quasiplane $\mathcal{P}$.

To check $F \circ H$ is bilipschitz, we let $x=\left(x_{1}, x_{3}\right), y=\left(y_{1}, y_{3}\right) \in \mathbb{R}^{2}$ and assume, as we may, that $\left|x_{1}\right| \geq\left|y_{1}\right|$. We consider four cases based on the relative locations of these points.

Case I. $\left|x_{1}\right|>0,\left|x_{1}-y_{1}\right| \leq\left|x_{1}\right| / 7$ and $\sqrt{\left|x_{3}-y_{3}\right|} \leq\left|x_{1}\right| / 7$. In this case, $\left|x_{1}\right| \simeq$ $\left|y_{1}\right|$, the Grushin distance $d_{\mathbb{G}}(x, y) \simeq\left|x_{1}-y_{1}\right|+\frac{\left|x_{3}-y_{3}\right|}{\left|x_{1}\right|}$ and the Euclidean distance $|H(x)-H(y)| \simeq\left|x_{1}\right|\left|x_{1}-y_{1}\right|+\left|x_{3}-y_{3}\right|$. Suppose $H(x)$ is in a rectangle $\mathrm{E}_{k, m}$, then $7^{k-1} \leq\left|x_{1}\right| \leq 7^{k}$ and $H(y)$ is in $\check{\mathrm{E}}_{k, m}$. Since $F \mid \check{\mathrm{E}}_{k, m}$ is a $\left(7^{-k}, C\right)$-quasisimilarity for some $C>1,|F \circ H(x)-F \circ H(y)| \simeq 7^{-k}\left(\left|x_{1}\right|\left|x_{1}-y_{1}\right|+\left|x_{3}-y_{3}\right|\right) \simeq d_{\mathbb{G}}(x, y)$. The proof is the same if $H(x)$ is in $\mathrm{E}_{k, m}^{\prime}$.

Case II. $\left|x_{1}\right|>0,\left|x_{1}-y_{1}\right| \geq\left|x_{1}\right| / 7$ and $\sqrt{\left|x_{3}-y_{3}\right|} \leq\left|x_{1}\right| / 7$. In this case, $d_{\mathbb{G}}(x, y) \simeq\left|x_{1}-y_{1}\right| \simeq\left|x_{1}\right|$ and $|H(x)-H(y)| \simeq\left|x_{1}\right|^{2}$. Suppose $H(x)$ is in $\mathrm{E}_{k, m}$, as before diam $\mathrm{E}_{k, m} \simeq 49^{k} \simeq\left|x_{1}\right|^{2}$. Since $F$ is quasisymmetric and $F \mid \mathrm{E}_{k, m}$ is a $\left(7^{-k}, C\right)$-quasisimilarity, $|F \circ H(x)-F \circ H(y)| \simeq \operatorname{diam} F\left(\mathrm{E}_{k, m}\right) \simeq 7^{k} \simeq d_{\mathbb{G}}(x, y)$. The proof is the same if $H(x)$ is in $\mathrm{E}_{k, m}^{\prime}$.

Case III. $\left|x_{1}\right|>0$ and $\sqrt{\left|x_{3}-y_{3}\right|} \geq\left|x_{1}\right| / 7$. In this case, $d_{\mathbb{G}}(x, y) \simeq \sqrt{\left|x_{3}-y_{3}\right|}$ and $|H(x)-H(y)| \simeq\left|x_{3}-y_{3}\right|$. Assume again $H(x)$ is in $\mathrm{E}_{k, m}$. After applying in $\mathbb{G}$ a translation $\left(z_{1}, z_{3}\right) \mapsto\left(z_{1}, z_{3}-m 49^{k}\right)$ followed by a dilation $\left(z_{1}, z_{3}\right) \rightarrow$ $\left(7^{-k} z_{1}, 49^{-k} z_{3}\right)$, we assume as we may that $H(x) \in \mathrm{P} \cap \mathrm{Q}$. Choose $j$ to be the smallest nonnegative integer $j$ such that $H(y) \in \zeta^{-j} \mathrm{Q}$, then $|H(x)-H(y)| \simeq 49^{j}$ and $d_{\mathbb{G}}(x, y) \simeq 7^{j}$. From the definition of $F$ it follows that $|F \circ H(x)-F \circ H(y)|=$ $\left|F_{j} \circ H(x)-F_{j} \circ H(y)\right| \simeq 7^{j}$, hence $|F \circ H(x)-F \circ H(y)| \simeq d_{\mathbb{G}}(x, y)$. The case $H(x) \in \mathrm{E}_{k, m}^{\prime}$ is the same.

Case IV. $x_{1}=0 .|F \circ H(x)-F \circ H(y)| \simeq d_{\mathbb{G}}(x, y)$ can be obtained by taking limits in Case III.

This shows that $F \circ H: \mathbb{G} \rightarrow \mathcal{P}$ is bilipschitz and completes the proof of Theorem 1.1. 


\section{Appendix}

We construct paths $J_{I}$ and $J_{L}$ in Section 2.2 following Assouad in [1]. To start, we subdivide the cube $\mathcal{C}=\left[-\frac{7}{2}, \frac{7}{2}\right] \times\left[-\frac{7}{2}, \frac{7}{2}\right] \times[0,7]$ into $7^{3}$ unit cubes. The centers of these subcubes are $\left(z_{1}, z_{2}, z_{3}\right)$, with $z_{1}, z_{2} \in\{-3,-2,-1,0,1,2,3\}$ and $z_{3} \in\left\{\frac{1}{2}, \frac{3}{2}, \frac{5}{2}, \frac{7}{2}, \frac{9}{2}, \frac{11}{2}, \frac{13}{2}\right\}$. Each of the 49 unit segments in $J_{I}$ (respectively $J_{L}$ ) will pass through the center of and is contained in one of these unit cubes.

We define a polygonal path $J^{\prime}$ by listing its starting point, its terminal point and all points along the path where it makes a turn, which will be 'half' of the final paths, as follows

$$
\begin{aligned}
J^{\prime}: & (0,0,0) \rightarrow\left(0,0, \frac{3}{2}\right) \rightarrow\left(0,1, \frac{3}{2}\right) \rightarrow\left(-2,1, \frac{3}{2}\right) \rightarrow\left(-2,-2, \frac{3}{2}\right) \\
& \rightarrow\left(2,-2, \frac{3}{2}\right) \rightarrow\left(2,0, \frac{3}{2}\right) \rightarrow\left(2,0, \frac{5}{2}\right) \rightarrow\left(2,-2, \frac{5}{2}\right) \\
& \rightarrow\left(-2,-2, \frac{5}{2}\right) \rightarrow\left(-2,0, \frac{5}{2}\right) \rightarrow\left(-1,0, \frac{5}{2}\right) \rightarrow\left(-1,0, \frac{7}{2}\right) .
\end{aligned}
$$

Clearly $J^{\prime}$ is a simple path; except for its end points, $J^{\prime}$ is contained in the intersection of the half spaces $x_{3}<\frac{7}{2}$ and $x_{3}<\frac{7}{2}-x_{2}$. Denote by $J^{\prime \prime}$ the reflection of $J^{\prime}$ with respect to the plane $x_{3}=\frac{7}{2}$, and by $J^{\prime \prime \prime}$ the reflection of $J^{\prime}$ with respect to the plane $x_{3}=\frac{7}{2}-x_{2}$. Both paths $J_{I}=J^{\prime} \cup J^{\prime \prime}$ and $J_{L}=J^{\prime} \cup J^{\prime \prime \prime}$ are simple.

Since the $x_{3}$-coordinate is monotone along path $J^{\prime}, J_{I}$ is unknotted in $Q_{I}$ and $J_{L}$ is unknotted in $Q_{L}$. Because $\left|x_{1}\right|+\left|x_{2}\right| \leq 2$ on $J^{\prime}$, the blocks associated to the 49 unit segments in $J_{I}$ (respectively $J_{L}$ ) are contained in $Q_{I}$ (respectively $Q_{L}$ ). Other properties required for paths $J_{I}$ and $J_{L}$ are obvious.

\section{References}

[1] P. Assouad, Plongements lipschitziens dans $\mathbf{R}^{n}$, Bull. Soc. Math. France 111 (1983), 429448.

[2] A. Bellä̈CHE, The tangent space in sub-Riemannian geometry, In: "Sub-Riemannian geometry", Progr. Math., Vol. 144, Birkhäuser, Basel, 1996, 1-78.

[3] M. BonK and J. Heinonen, Smooth quasiregular mappings with branching, Publ. Math. Inst. Hautes Études Sci. 100 (2004), 153-170.

[4] F. FERRARI and B. FRANCHI, Geometry of the boundary and doubling property of the harmonic measure for Grushin type operators, Rend. Sem. Mat. Univ. Politec. Torino (3) 58 (2002), 281-299. Partial differential operators (Torino, 2000).

[5] B. FRANCHI and E. LANCONELLI, Une métrique associée à une classe d'opérateurs elliptiques dégénérés, In: "Conference on Linear Partial and Pseudodifferential Operators" (Torino, 1982), Rend. Sem. Mat. Univ. Politec. Torino, Special Issue (1983), 105-114.

[6] W. MEYERSON, The Grushin plane and quasiconformal Jacobians, preprint, 2011.

[7] P. PAnsu, Métriques de Carnot-Carathéodory et quasiisométries des espaces symétriques de rang un, Ann. of Math. (2) 129 (1989), 1-60.

[8] S. SEMMES, On the nonexistence of bi-Lipschitz parameterizations and geometric problems about $A_{\infty}$-weights, Rev. Mat. Iberoam. 12 (1996), 337-410. 
[9] J. SEO, A characterization of bi-Lipschitz embeddable metric spaces in therms of local bi-Lipschitz embeddability, Math. Res. Lett. (6) 18 (2011), 1179-1202.

[10] J. VÄIsÄLÄ, "Lectures on $n$-dimensional Quasiconformal Mappings", Lecture Notes in Mathematics, Vol. 229, Springer-Verlag, Berlin, 1971.

Department of Mathematics

University of Illinois

1409 West Green Street

Urbana, IL 61822, USA

wu@math.uiuc.edu 